perspective, by sharing these catalog records in the national databases, we hope to integrate these items with the larger universe of related materials elsewhere, both manuscript and print, and hope that other libraries holding such manuscripts will join us in bringing these important but relatively under-researched primary materials to the attention of scholars in many fields.
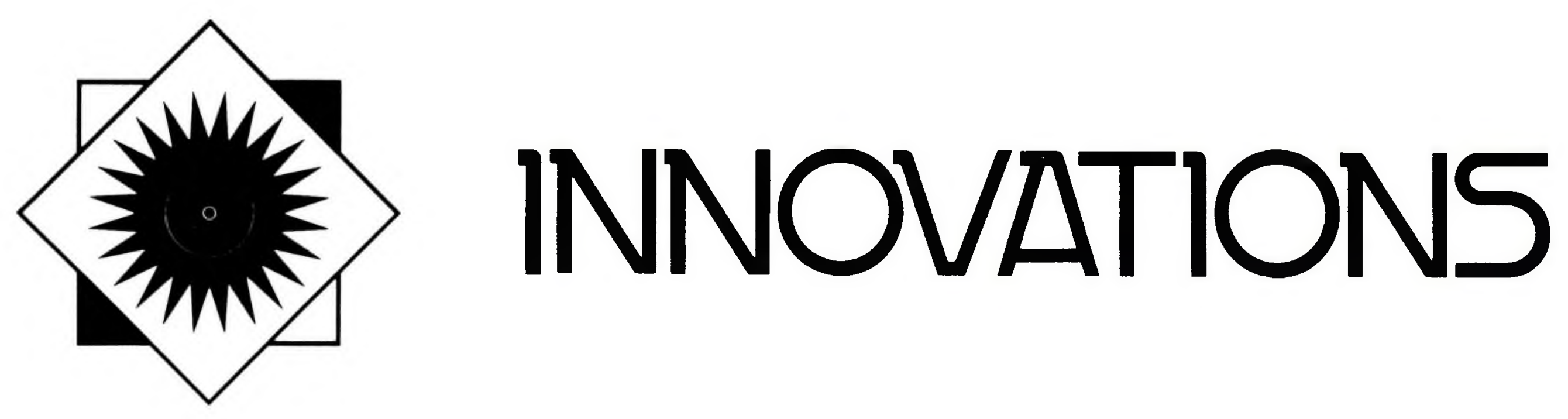

\title{
MAC MICRO: Solving the problems of microcomputer applications in an academic library
}

\author{
By Diane Richards \\ Reference Librarian \\ North Dakota State University Library*
}

Microcomputers have become a fact of everyone's life. In academic library work areas they have sprouted like weeds, to the great chagrin of the computer-phobic. Virtually no one has time to sit down and work through manuals. New versions of software come out with dizzying speed. How are harried librarians and staff to cope? One approach to the problem is being tried at Washington State University, where the Microcomputer Applications Committee (MAC) came into existence 18 months ago.

At the time of MAC's creation, every unit in the library was going its own way with regard to microcomputers. Hardware and software were purchased out of departmental budgets, in response to individual needs and often for specialized applications, producing an astonishing variety of configurations. Different units in the library often didn't know what was going on down the hall. Inevitably, there was much duplication of effort, particularly in training, and no uniformity in software. At least three different word processing packages were in use on three different floors, making shared data files an impossibility.

The creation of systems librarian Jim Kopp, MAC is designed to bring order out of this chaos. Major goals are to promote communication among

* The author recently moved to North Dakota State after working on the staff of the Washington State University Libraries. the different units with regard to microcomputers and to develop a core of expertise within the libraries. With this in mind, the composition of MAC consists of one representative from each of six units or divisions within the Library: Administration; Humanities/Social Sciences/Education; Technical Services; Science/Veterinary Medicine/ Agricultural Sciences; Instructional Media Services; and Manuscripts, Archives, and Special Collections. Membership is not limited to professional staff, but is open to anyone in the unit. One highly desirable qualification is microcomputer expertise, or, if that is not available, interest and a willingness to learn. In fact, when MAC convened for the first time, a wide range of skill was represented, reflecting in many respects the varying levels of microcomputer use among the library units. Given this variety, the sharing of information has become one of the primary functions of the meetings, with the frequent discovery that what is a problem in one unit has already been solved in another.

The first order of business for the Committee was to put together a library policy on microcomputers. This policy was intended to set out the rules governing such issues as software copyright and licensing, and ownership of databases created by library staff using library-owned hardware and software. The desirability of software standardization and training for all staff using microcomputers is another area covered. Much of the content of this policy formalizes practices already adopted. How- 
ever, the importance of having an actual document can hardly be overstated. The limits of library liability have been established and the rules are available for reference.

Another project undertaken by the Committee was an inventory of hardware and software owned by the units. As a result of this, real focus and consistency is possible when considering equipment needs. A later inventory focused on amount and type of usage, with the intent of ensuring optimum use of available machines. Although no machines were found to be underutilized, microcomputers could have been reassigned if necessary. Both of these inventories will be kept current.

Since word processing is one of the major applications for microcomputers at Washington State University Libraries, MAC felt standardization in software was essential. In addition to the problem of multiple packages, there were many different versions of the same software in use. Each had its adherents. The Committee decided to hold a demonstration, showing features of each of the main contenders. Relevant reviews were copied and cir- culated to the Committee members. Input was solicited within each unit from the secretaries and any others directly involved with word processing. The final decision was to standardize to Microsoft Word, with enough copies being purchased to ensure all units had access to the same version. With this decision, it has become possible within the libraries to share files on diskette, making collaboration much simpler. Since many other WSU departments have also standardized to Microsoft Word, interdepartmental communication has been facilitated. Library publications can be given to University Printing on diskette and typeset directly, saving a substantial amount of time and money.

As the second phase of MAC's existence begins, emphasis has shifted to training. In-house seminars have begun on using Microsoft Word, and a users' group for this software is contemplated. The Committee can feel assured that it has made a good beginning in bringing some order to the chaotic world of microcomputers for the faculty and staff of the Washington State University Libraries.

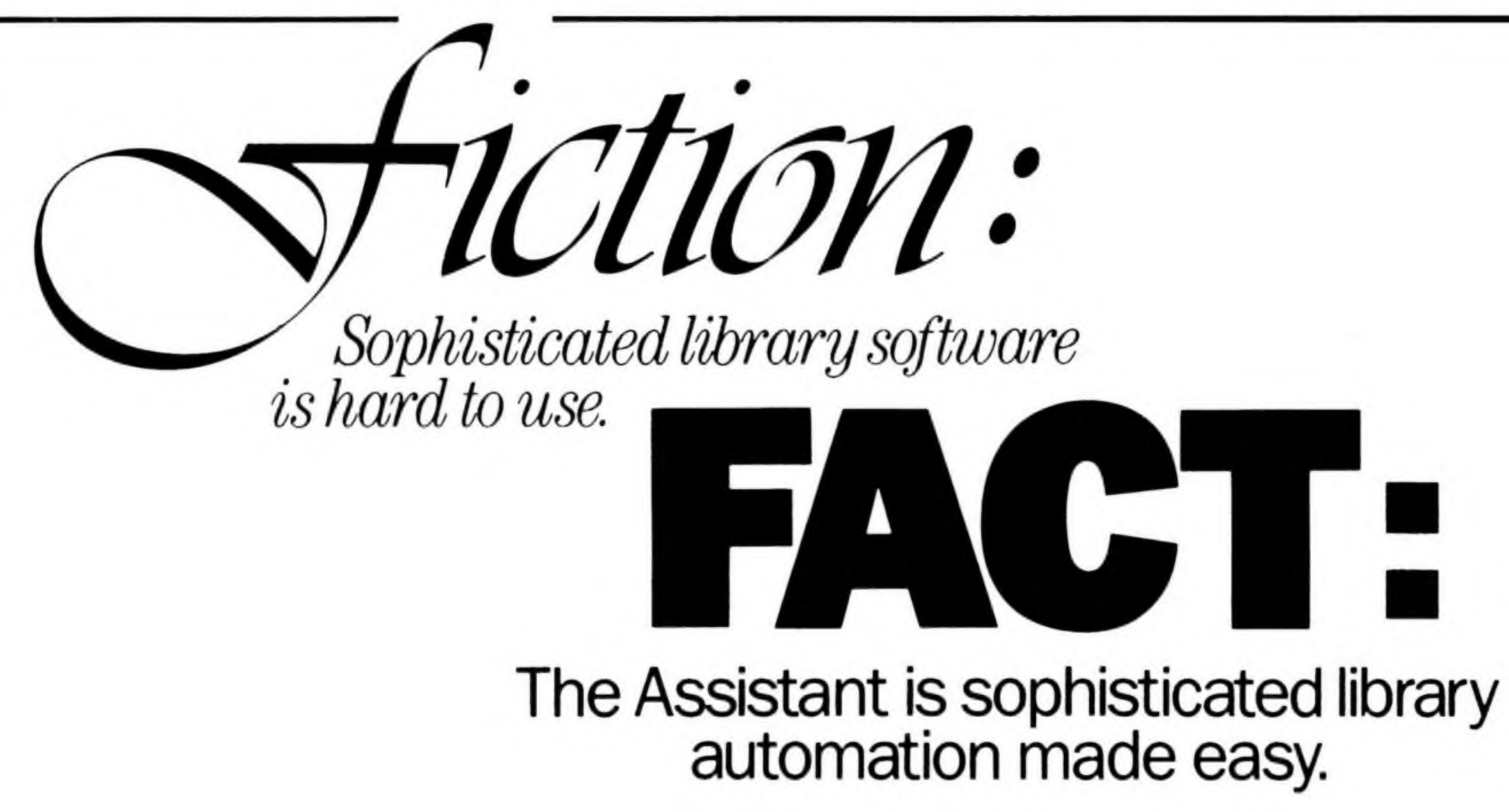

At last, a complete automation software package that's genuinely easy to use. The Assistant is sophisticated enough to meet your every need-and so manageable that little or no training is required. Developed with the small to medium-sized library in mind, The Assistant offers modules to handle:

- Circulation Control - Cataloging

- Serials Control - MARC Interface

- Acquisitions/Accounting
Our product is so well-designed you'll wonder how you ever got along without it. And we stand behind every system with a complete client support package-including daily telephone access, if needed.

The Assistant is not only easy to use, its low price will surprise you. Call today for more facts about sophisticated library automation made easy. 